\title{
Effect of Different Porosity Ratio on Mechanical Properties of Polycaprolactone and Polylactic Acid Scaffolds
}

\author{
${ }^{1}$ Adem Demir, *2Mustafa Keser, and ${ }^{1}$ Fatih Caliskan \\ ${ }^{1}$ Faculty of Technology, Department of Metallurgical an Materials Engineering, Sakarya University of Applied Science, \\ Turkey \\ *2Nanoscience \& Nanoengineering Ph.D. Program, Sakarya University, Turkey \\ ${ }^{1}$ Faculty of Technology, Department of Metallurgical an Materials Engineering, Sakarya University of Applied Science, \\ Turkey
}

\begin{abstract}
In recent years, patient-specific solutions and additive manufacturing (AM) have become increasingly important in the treatment of bone defects in studies performed on the medical field. In this direction, additive manufacturing methods use in scaffold fabrication, and many advantages of these systems come to the forefront. Porosity affects the mechanical properties, biocompatibility, and biodegradability of tissue engineering scaffolds. In this study, the effect of different porosity ratios on the mechanical properties of scaffolds for polylactic acid (PLA) and polycaprolactone (PCL) scaffolds was studied. With this fabrication method can be formed entirely three dimensional (3D) interconnected porous scaffolds with pore size. Three different $(20 \%, 35 \%$, and $50 \%)$ porosity ratios were determined for both materials, and the mechanical properties of the samples were determined by compression test. The scaffolds fabricated with larger pore size showed lower mechanical performance compared to scaffolds with smaller pore size.
\end{abstract}

Keywords: Additive Manufacturing, Porosity Ratio, Polycaprolactone, Polylactic acid, Mechanical Properties, Tissue Engineering Scaffolds

\section{Introduction}

Scaffolds may be potentially metallic, ceramic, polymeric, natural or composite materials. Scaffolds are usually porous up to a ratio, but may also be non-porous[1]. The function of the tissue engineering scaffolds is to direct the development of cells by allowing the cells to migrate from the surrounding tissue and to assist in cell development within the hollow structure of the scaffold.[2]. Scaffolds with appropriate 3D microstructure and biomechanical properties support the promotion, development, reproduction and functional differentiation of cell attaching[3]. An ideal scaffold must have a 3D and highly interconnected hollow structure, have a biocompatible and bioresorbable structure, have a suitable surface structure for cell bonding, and differentiation, as well as mechanical properties compatible with the tissue to be applied.[4].

Biocompatibility, bioactivity, mechanical strength and porosity are some of the main features of scaffolding. These requirements can be obtained using biomaterials and AM processes[5]. The Fused Deposition Modeling process leaves a molten material fiber, usually made with a plastic filament, on a layer using a moving head. The material is heated to a temperature slightly above the melting point in the head, then extruded from a nozzle to a layer and cooled until it solidifies and forms a layer.[6].

PLA is semicrystalline, biodegradable, biocompatible, and has found use in several medical applications like orthopedic implants, drug delivery systems, and biofabrication[7]. PCL is a 
biodegradable polymer and is widely used to manufacture tissue scaffolds due to its good biocompatibility and excellent mechanical properties[8].

PLA and PCL have different physical properties and biocompatibility. PLA is an aliphatic polyester. PLA has a melting point of $170^{\circ} \mathrm{C}$, a glass transition temperature of $56^{\circ} \mathrm{C}$ and a crystallinity of up to $40 \%$. The PCL has a glass transition temperature of $-60{ }^{\circ} \mathrm{C}$ and a melting temperature of about 59-64 ${ }^{\circ} \mathrm{C}$. PCL has a slow deterioration rate (up to 2 years) and a high permeability; Therefore, it is very suitable for long-term implants and drug delivery systems[2].

Scaffolds were produced using polylactic acid, and polycaprolactone materials and scaffolds which have three different porosity ratios $(20 \%, 35 \%, 50 \%)$ were fabricated by using Fused Deposition Modeling process. The morphology and porosity dimensions of the produced scaffolds were examined by optical microscope. Mechanical properties of the scaffolds were determined by compression test.

\section{Material and Method}

\subsection{Material}

For PLA scaffolds, ready to use $1.75 \mathrm{~mm}$ diameter filament was used as a material for the fabrication of scaffolds, and PCL scaffolds, $1.75 \mathrm{~mm}$ diameter filament was fabricated from PCL pellets via extrusion process for PCL material, and scaffolds were fabricated with these filaments.

\subsection{Fabrication and Morphology of Scaffolds}

Scaffolds were fabricated via RepRap Prusa i3 3D printer. For the gcode and parameters required for the system, Slic3r program was used. In order to obtain a $300 \mu \mathrm{m}$ strand diameter in the fabrication of scaffolds, nozzle diameter and layer height were determined as $300 \mu \mathrm{m}$. Printing speed and temperature values were identified as $2000 \mathrm{~mm} / \mathrm{min}$ and $200{ }^{\circ} \mathrm{C}$ for PLA scaffolds, for PCL scaffolds printing speed and temperature values, were defined as $1200 \mathrm{~mm} / \mathrm{min}$ and 95 ${ }^{\circ} \mathrm{C}$. Scaffolds which were fabricated for morphology examination are fabricated with dimensions of $10 \times 10 \times 5 \mathrm{~mm}$.

For the compression test, the scaffolds were fabricated with dimensions of $10 \times 10 \times 20 \mathrm{~mm}$. Porosity ratio of PLA and PCL scaffolds were determined as 20\% (PLA20, PCL20), 35\% (PLA35, PCL35), and 50\% (PLA50, PCL50) with printing software.

Zeiss Axio Lab A1 optical microscope was used to examine the morphology of the scaffolds. The cross-sectional measurements of the scaffolds were investigated with AxioVision software and strand, and porosity dimensions were measured. 


\subsection{Mechanical Testing}

The mechanical test of the scaffolds was examined by compression test. Mechanical properties of the scaffolds were obtained by using the Shimadzu 50KN table-top model test system. Data from the system is processed with Trapezium X material testing software. With a size of $10 \times 10$ x $20 \mathrm{~mm}$ scaffolds were tested to be five samples per group, test parameters were determined as the speed of $1.3 \mathrm{~mm} / \mathrm{min}$ and $2 \mathrm{KN}$ cell load. The compressive modulus was determined based on the slope of the linear region of the stress versus strain curve. Compressive strength was determined to calculate by dividing the maximum compressive load applied by the specimen during the test by the original minimum cross-sectional area of the specimen.

\section{Results And Discussion}

\subsection{Fabrication and Morphology of Scaffolds}

Fig. 1 and Fig. 2 show the morphology images of PLA and PCL scaffolds fabricated. Porosity size of fabricated scaffolds for PLA50, PLA35, and PLA20, were measured as respectively, 302 $\mu \mathrm{m}$ to $313 \mu \mathrm{m}, 188 \mu \mathrm{m}$ to 164 and 98 to 92 (Fig. 1).

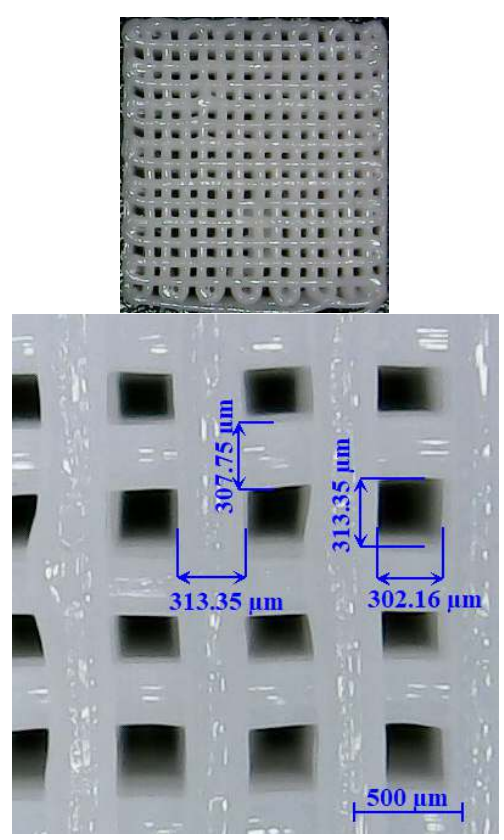

(a)

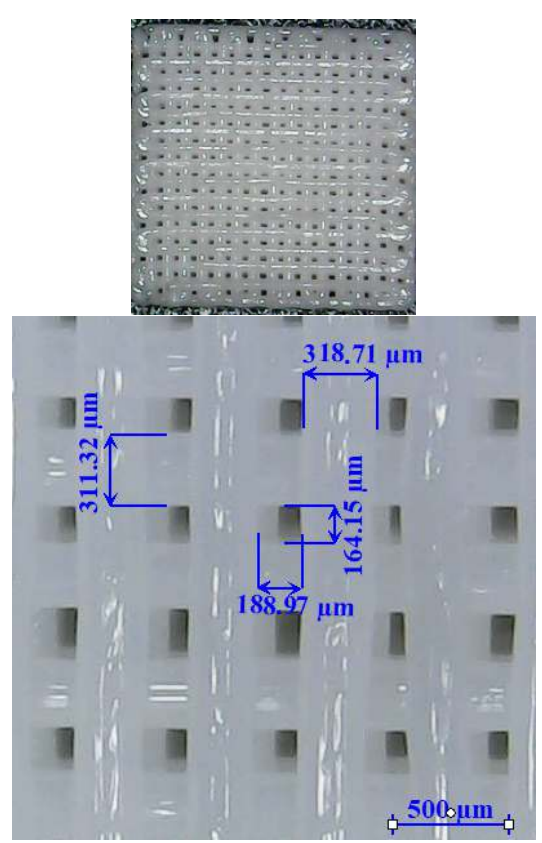

(b)

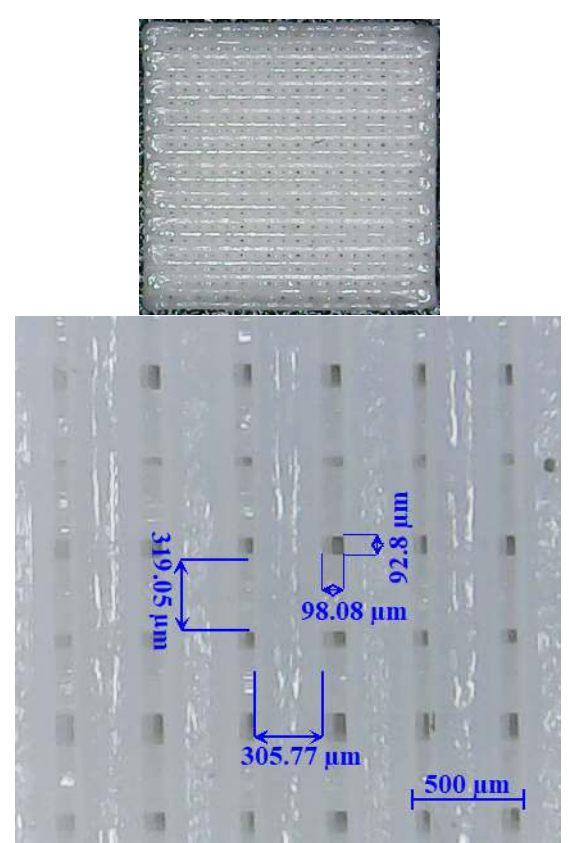

(c)

Figure 1. Optical microscope images of PLA scaffolds, a) PLA50, b) PLA35 and c) PLA20.

Porosity size of fabricated scaffolds for PCL50, PCL35, and PCL20 were measured as respectively, $305 \mu \mathrm{m}$ to $324 \mu \mathrm{m}, 158 \mu \mathrm{m}$ to $162 \mu \mathrm{m}$ ve $73 \mu \mathrm{m}$ to $97 \mu \mathrm{m}$ (Fig. 2). As for the strand diameters, it has been observed that both materials change within a range of from $290 \mu \mathrm{m}$ to $334 \mu \mathrm{m}$ (Fig. 1 and fig. 2). The cause for the strand diameters below $300 \mu \mathrm{m}$ was determined by the elongation caused by printing over the pore region of the new strand during the fabrication 
of the scaffold. It was determined that strand diameters larger than $300 \mathrm{~mm}$ be caused by extrusion deformation and printing of new strand to none pore size region.

As a result of the morphological examination, it was determined that the scaffolds were produced successfully in both materials and there was no network and strand disconnection in the scaffold.

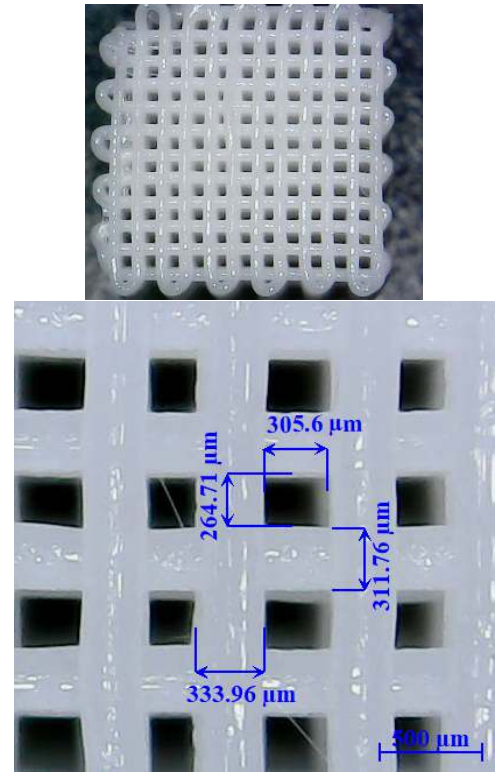

(a)

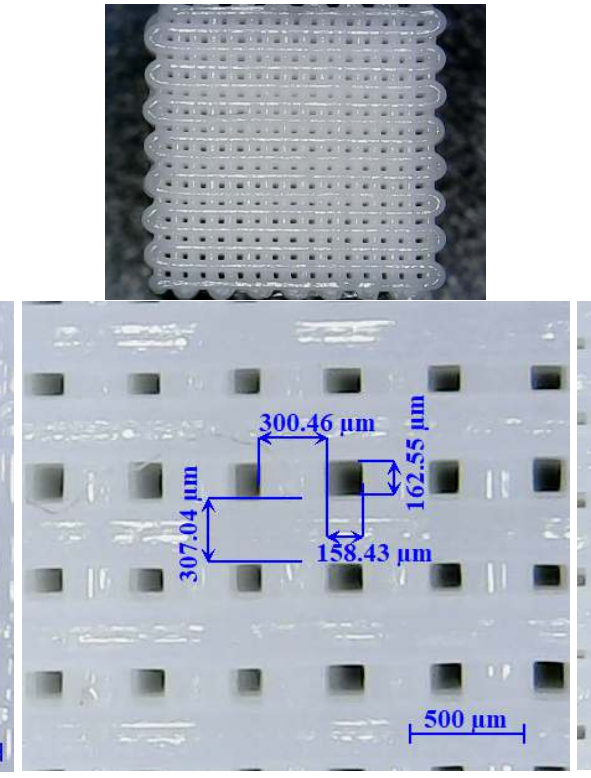

(b)
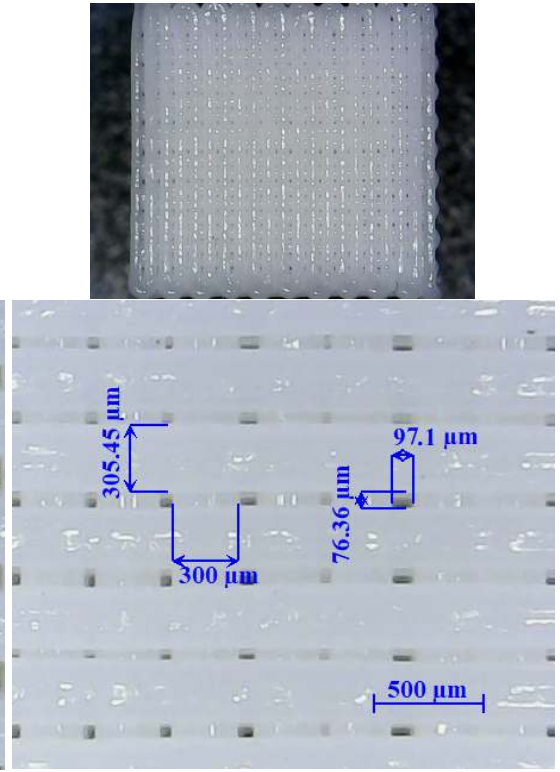

(c)

Figure 2. Optical microscope images of PCL scaffolds a) PCL50, b) PCL35, and c) PCL20.

\subsection{Mechanical Testing}

Preferably, a scaffold should have the one of a characteristic which is mechanical properties to equivalent those of the tissues at the site of implantation[4]. In addition to mechanical properties, scaffolds must provide appropriate pore size distribution for the transport of nutrients and wastes. [9]. Fig. 3 shows the stress-strain curves of PLA and PCL scaffolds. Stress-strain curves showed linearity regions where each scaffold group exhibited elastic behavior.

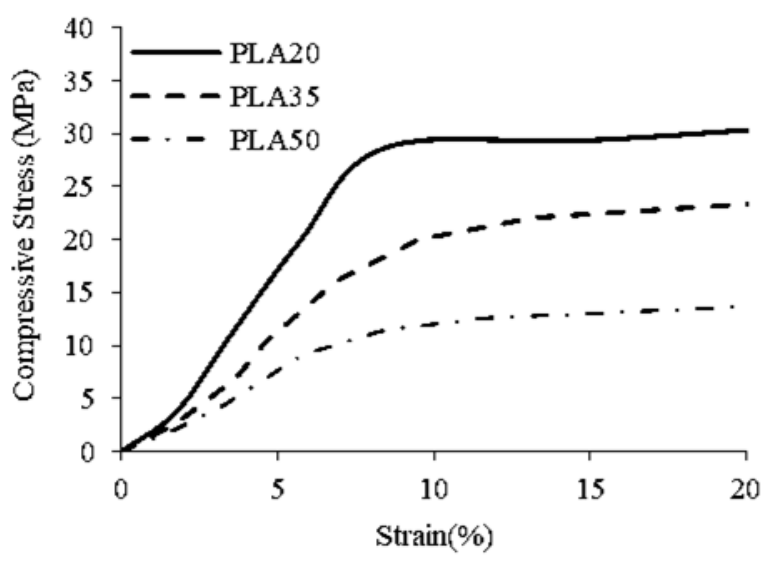

(a)

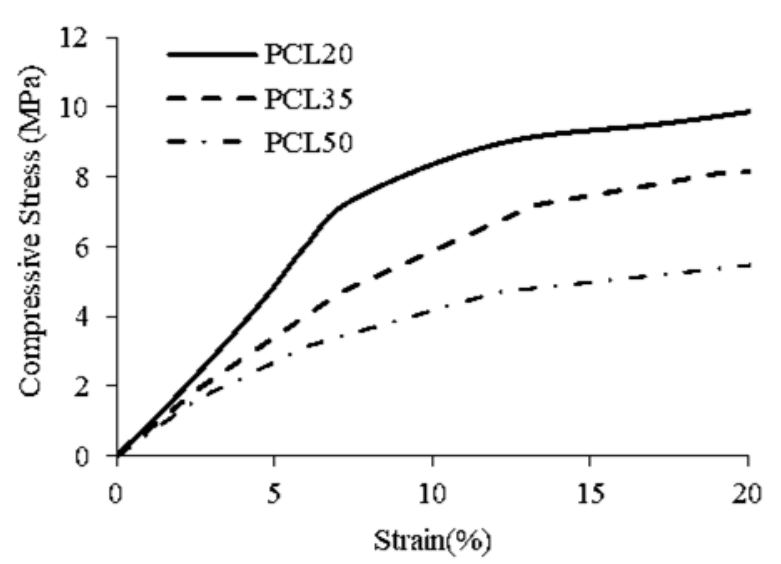

(b)

Figure 3. Stress-Strain curves: a) Stress-strain curves of PLA20, PLA35, and PLA50 scaffolds, b) Stress-strain curves of PCL20, PCL35, and PCL50 scaffolds. 
As the amount of porosity increased in both materials, respectively, the compression stress, the slope of the curves decreased, the elasticity increased and the lowest mechanical performances were observed in PLA50 and PCL50 scaffolds.

As compressive strength; In parallel with the elastic behavior of the scaffold groups, the compressive strength was increased as the pore rate decreased, and the maximum compressive strengths were $79 \mathrm{MPa}$ for PLA20 and $38 \mathrm{MPa}$ for PCL20 when both material groups were evaluated among themselves (Figure 4).

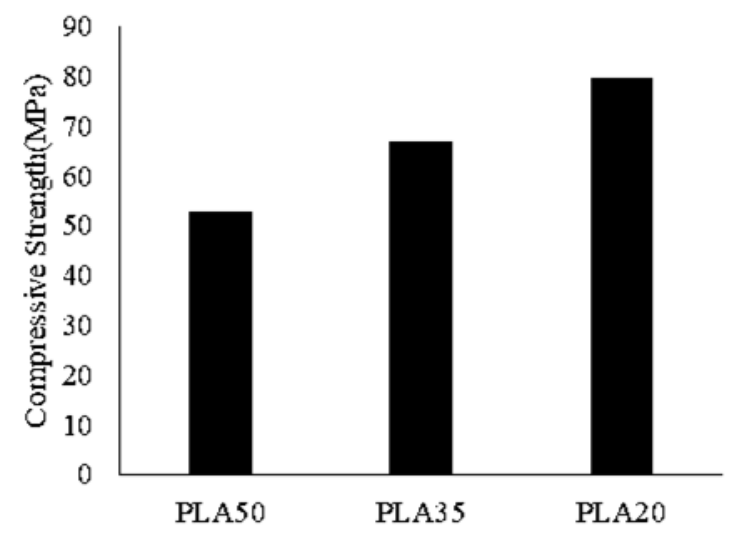

(a)

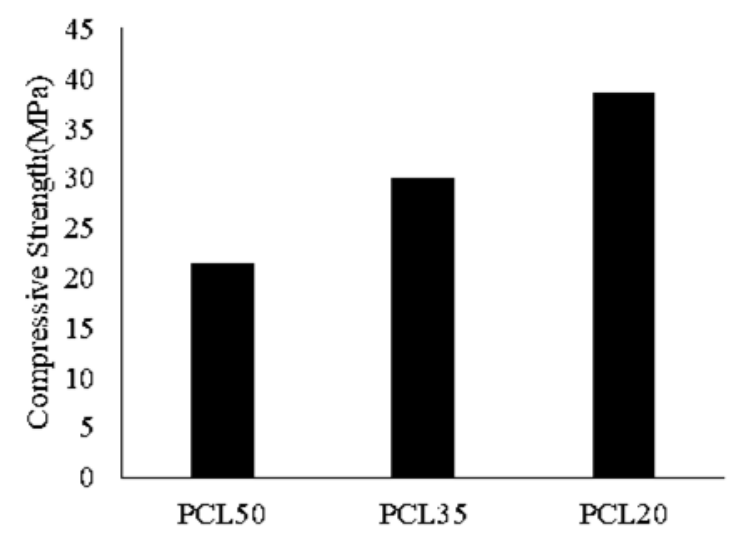

(b)

Figure 4. Compression strengths: a) compressive strengths of PLA20, PLA35, and PLA50 scaffolds; b) compressive strengths of PCL20, PCL35, and PCL50 scaffolds.

\section{Conclusions}

In this study, scaffolds which have different porosity ratios were fabricated by using PLA and PCL materials with using FDM process, and the effect of porosity ratio on the mechanical properties of scaffolds was discussed. Scaffolds were fabricated with $20 \%, 35 \%$, and $50 \%$ porosity ratio. As a result of the investigation of the scaffolds' morphology via an optical microscope, it is determined that scaffolds are highly porous and interconnected. As a result of the compression tests, it has been observed that the compressive strength of scaffolds decreases as the porosity ratio increases and it shows higher percent elongation at the stress per unit. 


\section{References}

[1] ASTM, E. I. Materials, P. Under, A. Service, C. Plastics, and T. A. Prop-, "Standard Guide for Characterization and Testing of Biomaterial Scaffolds Used in TissueEngineered Medical Products," F2150-13, pp. 1-10, 2013.

[2] A. Kramschuster and L. Turng, "Fabrication of Tissue Engineering Scaffolds," in Handbook of Biopolymers and Biodegradable Plastics, Elsevier, 2013, pp. 427-446.

[3] V. Tran and X. Wen, "Rapid prototyping technologies for tissue regeneration," in Rapid Prototyping of Biomaterials, Elsevier, 2014, pp. 97-155.

[4] D. W. Hutmacher, "Scaffolds in tissue engineering bone and cartilage," in The Biomaterials: Silver Jubilee Compendium, vol. 21, no. 24, Elsevier, 2006, pp. 175-189.

[5] T. Özel, P. J. Bártolo, E. Ceretti, J. D. De Ciurana Gay, C. A. Rodríguez, and J. V. L. Da Silva, Biomedical devices: Design, prototyping, and manufacturing. 2016.

[6] N. Guo and M. C. Leu, "Additive manufacturing: Technology, applications and research needs,” Front. Mech. Eng., vol. 8, no. 3, pp. 215-243, 2013.

[7] J. K. Carrow, P. Kerativitayanan, M. K. Jaiswal, G. Lokhande, and A. K. Gaharwar, "Polymers for Bioprinting," in Essentials of 3D Biofabrication and Translation, Elsevier, 2015, pp. 229-248.

[8] J. S. Park et al., "Fabrication and characterization of 3D-printed bone-like $\beta$-tricalcium phosphate/polycaprolactone scaffolds for dental tissue engineering,” J. Ind. Eng. Chem., vol. 46, pp. 175-181, Feb. 2017.

[9] R. Narayan, T. Boland, and Y. Lee, Printed Biomaterials - Novel Processing and Modeling Techniques for Medicine and Surgery. 2010. 